\title{
Examining the cost effectiveness of interventions to promote the physical health of people with mental health problems: a systematic review
}

\author{
A-La Park ${ }^{1 *}$, David McDaid ${ }^{1,2}$, Prisca Weiser ${ }^{3}$, Carolin Von Gottberg ${ }^{3}$, Thomas Becker ${ }^{4}$, Reinhold Kilian ${ }^{4}$
} and for the HELPS Network

\begin{abstract}
Background: Recently attention has begun to focus not only on assessing the effectiveness of interventions to tackle mental health problems, but also on measures to prevent physical co-morbidity. Individuals with mental health problems are at significantly increased risk of chronic physical health problems, such as cardiovascular disease or diabetes, as well as reduced life expectancy. The excess costs of co-morbid physical and mental health problems are substantial. Potentially, measures to reduce the risk of co-morbid physical health problems may represent excellent value for money.
\end{abstract}

Methods: To conduct a systematic review to determine what is known about economic evaluations of actions to promote better physical health in individuals identified as having a clinically diagnosed mental disorder, but no physical co-morbidity. Systematic searches of databases were supplemented by hand searches of relevant journals and websites.

Results: Of 1970 studies originally assessed, 11 met our inclusion criteria. In addition, five protocols for other studies were also identified. Studies looked at exercise programmes, nutritional advice, smoking, alcohol and drug cessation, and reducing the risk of blood borne infectious diseases such as HIV/AIDS and hepatitis. All of the lifestyle and smoking cessation studies focused on people with depression and anxiety disorders. Substance abuse and infectious disease prevention studies focused on people with psychoses and bipolar disorder.

Conclusions: There is a very small, albeit growing, literature on the cost effectiveness of interventions to promote the physical health of people with mental health problems. Most studies suggest that value for money actions in specific contexts and settings are available. Given that the success or failure of health promoting interventions can be very context specific, more studies are needed in more settings, focused on different population groups with different mental health problems and reporting intermediate and long term outcomes. There is a need to better distinguish between resource use and costs in a transparent manner, including impacts outside of the health care system. Issues such as programme fidelity, uptake and adherence should also be accounted for in economic analysis. The role of behavioural psychological techniques to influence health behaviours might also be considered.

Keywords: Health promotion, Mental health, Economic evaluation, Co-morbidities, Public health

\footnotetext{
* Correspondence: A.Park@lse.ac.uk

${ }^{1}$ Personal Social Services Research Unit, London School of Economics and

Political Science, London, UK

Full list of author information is available at the end of the article
} 


\section{Background}

Protecting the physical health of people with mental disorders is becoming more prominent in national and regional mental health policies [1-3]. This policy interest comes at a time when a growing number of studies have demonstrated that people with mental disorders are more likely to have costly co-morbid physical health problems than would be seen in the general population [4-7]. Illness, such as psychoses, bipolar disorder and major depression increase the risk of diseases such as obesity, diabetes, cardiovascular disease and chronic obstructive pulmonary disorder $[8,9]$.

Several factors can contribute to poor physical health. Physical illness such as cardiovascular disease, diabetes mellitus and chronic obstructive pulmonary disorder can go under reported and under treated in people with mental health problems [10-12]. Individuals may be reluctant to come into contact with health care services for fear of being labelled as having mental health problems, while clinicians may not place enough emphasis on the management of physical health and provide inadequate assessment, monitoring and care. For instance, one previous review reported that screening rates for metabolic syndrome in people with severe mental health problems remains low [10]. Negative attitudes in some health care professionals may also mean that some physical symptoms are wrongly thought to be a symptom of mental illness rather than an indication of a physical illness [13]. In one recent survey of nearly 800 people living with schizophrenia in 27 European countries, $17 \%$ felt that they experienced discrimination when treated for physical health problems [14].

There may also be a lack of incentives in primary care to monitor physical health problems, while the organisation of secondary health care systems in some countries, where mental health services may be largely separated from physical health services, can compound these issues, making the provision of seamless care for both physical and mental health problems more difficult to achieve. The association between poverty and poor mental health may also increase the likelihood that those with more severe mental health problems may live in areas of socioeconomic deprivation, which could further impact on their access to and utilisation of health care services

Poor lifestyle behaviours also remain critical contributory factors. This is particularly important given that there may be adverse effects of some medications prescribed to people with mental health problems. Rates of smoking in people with mental health problems are typically much higher than those seen in the general population. In one English population survey, for example, $42 \%$ of all cigarettes were smoked by people with mental disorders in 2007 [15]. Reviews covering a range of mental health problems in different population groups in different settings have also highlighted lower levels of physical activity, often combined with poor eating habits and nutritional intake [16-22].

There may also be increased risks of communicable disease such as sexually transmitted infections, including HIV/AIDS, as well as blood borne conditions including hepatitis because of cognitive dysfunction, multiple casual sex partners and unsafe needle sharing among some individuals who also have substance abuse problems $[23,24]$. The increased risk of homelessness, temporary accommodation or living in institutional settings also compounds the risks of both non communicable and infectious diseases [25,26].

These and other co-morbid physical health problems increase the risk of mortality compared with the general population. This difference in life expectancy can be stark. A study of men and women with severe mental disorders in Denmark, Finland and Sweden reported that they lived 20 and 15 years less respectively than the general population [27]. In an analysis of case registry data in London, and compared with the general population, women with schizophrenia, schizoaffective disorder or bipolar disorder lost between 9.8 and 17.5 years of life, while men lost between 8.0 and 14.6 years [28]. Life expectancy was also lower for individuals in this registry who were being treated for depressive disorders - with a reduced life expectancy of 10.6 and 7.2 years for men and women compared to the general population. Using data from the UK General Practice Research Database on 46,000 people with severe mental illness, Osborn and colleagues also reported a threefold difference in risk of cardiovascular deaths for those between 18 and 49 and an almost twofold increased risk for those aged 50-75 [29].

There is also evidence to suggest that inequalities in all causes of mortality risk appear to have widened between those with severe mental health problems and the general population. One meta-analysis looking at schizophrenia reported a significant increase in standardised mortality ratios using data from twenty-five countries over the period from 1980 to 2006, despite the increased availability of medications that can help individuals better manage mental health problems [30].

Co-morbidity has also been associated with substantially increased economic burden. It compounds the adverse impacts of mental illness with excess costs for the treatment for physical health problems [31-37]. It can also increase the likelihood that individuals will not be able to participate in the labour force, education or look after their families.

All of these factors emphasise the importance of identifying effective approaches to promote and protect the physical health of people with mental health problems, taking into account behavioural, environmental and iatrogenic health risks [38]. Recent reviews indicate an 
increasing literature on clinical effectiveness studies of interventions to promote physical health and/or treat comorbid physical health problems in people with mental health problems [39-42].

Potentially measures that are effective in reducing the risk or consequences of co-morbid physical health problems may represent excellent value for money. While there has been some economic analysis of interventions to promote and protect mental health [43], to date comparatively little attention appears to have been paid to the cost effectiveness of physical health promoting interventions for this population. Given that health care budget holders have to make difficult choices on how to allocate scarce resources to mental health and other services, it is crucial to know whether any investment in interventions/programmes to prevent physical health problems in people with mental health problems represents a worthwhile action. What, for instance, are the consequences for resource use in both the health care and related sectors?

\section{Objectives}

Given the importance of this topic the aim of this paper is to conduct a systematic review to identify economic evaluations of interventions intended specifically to promote better physical health and/or prevent physical health problems in people with clinically diagnosed mental disorders. To date only one review appears to have included economic considerations in this area; focused on the effectiveness of psycho-educational, behavioural/exercise/diet modification interventions for people with severe mental disorders it was unable to find any cost-effectiveness studies [41].

\section{Methods}

A detailed review protocol for this analysis is available from the corresponding author on request. This has been developed in line with the established Preferred Reporting Items for Systematic Reviews and Meta Analyses (PRISMA) reporting guidelines [44]. A PRISMA checklist is also provided in Additional file 1.

\section{Inclusion and exclusion criteria Types of participants}

Human participants of any age from studies taking place in high income countries. These studies needed to look at individuals with a primary diagnosis of moderate or severe mental illness, including schizophrenia, bipolar disorder, moderate and major affective disorder and delusional disorder. We only included individuals with alcohol or substance abuse disorders where these were part of a dual diagnosis with one of these mental health problems. We also excluded diagnoses of Alzheimer's Disease and related disorders, eating disorders and intellectual disabilities, from our analysis.
As our focus here was on disease prevention and health promotion; we excluded studies that looked at the economic benefits of treating physical health problems in people with pre-existing mental and physical co-morbidities. Nor did we look at the economic literature on interventions to prevent mental health problems in people with chronic physical illness such as diabetes or cardiovascular disease. There were no other restrictions on socioeconomic or clinical characteristics.

\section{Types of intervention}

Any non pharmacological interventions specifically targeted at promoting the physical health of individuals living with mental health problems. Interventions could for instance include exercise programmes, nutritional advice, smoking, alcohol and drug cessation and infectious diseases prevention. Our analysis was restricted to interventions delivered in countries defined by the World Bank as being high income.

\section{Types of outcome measure}

We included all of the standard economic evaluation methods that have been defined in health economics [45]. In brief, all measure costs in the same way but differ in how they measure economic outcomes. Cost-effectiveness (CEA) and cost-consequences (CCA) analyses report outcomes using natural clinical units e.g. changes in symptom free days; cost-utility analyses (CUA) studies measure outcomes in a common metric either in terms of quality or disability adjusted life years gained; while cost-benefit analyses (CBA) elicit monetary values from the public for different health outcomes. In addition we also included cost-offset analyses (COA), which highlight the potential resource savings of an action without reporting on changes in health outcomes. Studies that looked at economic incentives such as monetary rewards or changes in the price of goods to promote behaviour change were also included within the scope of our review.

\section{Types of study}

All of the economic evaluations described above that bring together cost data with outcome data were eligible for inclusion regardless of whether they were performed prospectively alongside a controlled trial or other quasiexperimental study design or retrospectively using data from a previous effectiveness study. Economic modelling studies that estimated the potential cost effectiveness of interventions by synthesising evidence from trials and other data sources on effectiveness and costs were also eligible. We also recorded study protocol papers reporting current evaluations in order to help identify the current interest in this area, and the way in which the evidence base is likely to develop further. 


\section{Search process}

We designed detailed specific systematic search strategies for several health and social science bibliographic databases: PubMed, PsycINFO, CINAHL, Francis, SocIndex and EconLit, covering the period January 1990 to December 2012, with searches run for the period until June 2012 on 7 September 2012, and updated searches for the final six months of the 2012 run on 2 January 2013. Our search strategy for the PubMed database is provided in Additional file 2 as an example of search strategies used. We combined a wide range of phrases for mental disorders with health promotion and public health terms along with terms for economic evaluations and/or specific economic terms / phrases such as cost-benefit. Papers could be in any language, but they needed to have an English language abstract to be potentially eligible for inclusion.

Our electronic search was supplemented by handsearches of a small number of relevant journals. A limited search of Google Scholar was also undertaken, alongside scrutiny of relevant websites including thinktanks, university economic research groups and some government departments. We also looked at actual examples of programmes and guidelines for the promotion of physical health that were identified separately within our project [39].

References were initially imported into Endnote X5 and duplicates eliminated. Remaining references were initially checked independently by two reviewers, based on their title and abstract to decide if they met our inclusion criteria. In the case of disagreement, further discussions about inclusion/exclusion were made. Full texts of potential matching articles were retrieved and then assessed to determine whether they looked at both the costs and effectiveness of interventions Articles were excluded if full texts did not provide cost or resource data. Data on bibliographic information, the intervention and comparator, duration of study, target population, economic evaluation methods used, empirical study design, cost and resource findings, effectiveness results and synthesis of costs and effects were then extracted from eligible studies into a bespoke Excel data extraction form. We conducted a narrative analysis and review of these studies. We did not plan or conduct statistical meta-analysis or other formal, aggregative synthesis of the results of included studies. All costs were converted to 2010 International Dollars, as well as being reported in their original currency and price year in Table 1.

\section{Results}

As Figure 1 indicates, our search process resulted in 1970 references being identified. 50 articles were subsequently retrieved based on the relevance of their abstracts. Of these, six were excluded as they focused on interventions to improve co-morbid mental disorders for people with pre-existing physical disease, which was outside the scope of our current review. 27 studies which initially appeared to be relevant were eventually excluded as no actual detailed costing and resource use was reported and another was excluded because of a lack of information on any comparator group. This left 11 completed studies which are summarised in Table 1. Table 2 summarises information on a further five protocol papers for ongoing or planned economic evaluations that were identified and which should meet our inclusion criteria when completed.

Of completed studies seven were from the USA, with three from the UK and one from Spain. Seven studies focused on psychosis and/or bipolar disorder. Three of these studies focused on interventions to tackle substance misuse and its consequences in people with dual diagnosis, while four reported on infectious disease reduction strategies. The remaining four studies focused predominantly on people with depression and/or anxiety disorders, looking at lifestyle modification interventions in terms of a smoking cessation programme, as well as three programmes focused on the promotion of physical activity.

Looking at the type of economic evaluation technique used, five studies were cost-consequences analyses, with five cost-utility analyses and one cost-effectiveness analysis. No cost benefit analyses were identified. Eight of these economic evaluations were performed prospectively alongside randomised controlled trials. One of two retrospective studies used a non-randomised quasi-experimental study design to look at the adoption of the rehabilitative treatment facilities for military veterans [46]. Two studies explored the costs and benefits of treating co-morbid HIV infections by making use of modelling techniques $[47,48]$. We now look at these studies in more detail.

\section{Lifestyle-based interventions to promote physical health}

One key concern focuses on promoting a sustained increase in physical activity and improved nutrition among people with mental health needs. We identified three studies looking at different physical activity interventions. One Spanish study evaluated a walking-based, supervised exercise programme for low-income older women with moderate depression and/or obesity [49]. 55 women were randomly allocated to a 50 minute supervised walking programme, three times per week for six months, in addition to dietary advice plus best primary care alone, compared with 51 women receiving usual primary care and a recommendation to exercise. Outcomes examined included changes in mean Body Mass Index (BMI) and changes in quality of life. After six months mean BMI decreased from 29.7 to 29.4 in the walking group, but increased from 30.6 to 30.8 in the usual care group $(\mathrm{p}<0.003)$. Supervised walking was highly cost effective at $\$ 449$ (€311, 2005 prices) per QALY gained, with a 99.9\% probability of being cost effective assuming a cost per 
Table 1 Economic evaluations alongside empirical studies for interventions promoting physical health for people with mental health problems

\begin{tabular}{|c|c|c|c|c|c|c|c|}
\hline $\begin{array}{l}\text { Author, } \\
\text { Year of } \\
\text { Publication, } \\
\text { Country of } \\
\text { Study }\end{array}$ & $\begin{array}{l}\text { Intervention (I) } \\
\text { Comparator (C) }\end{array}$ & $\begin{array}{l}\text { Target Population } \\
\text { Duration of study }\end{array}$ & $\begin{array}{l}\text { Study Design } \\
\text { Type of analysis }\end{array}$ & $\begin{array}{l}\text { Summary of main } \\
\text { resource and cost results }\end{array}$ & $\begin{array}{l}\text { Physical health related } \\
\text { effectiveness results }\end{array}$ & $\begin{array}{l}\text { Perspective/ } \\
\text { Price year }\end{array}$ & $\begin{array}{l}\text { Synthesis of costs } \\
\text { and effects }\end{array}$ \\
\hline $\begin{array}{l}\text { Barnett et al. } \\
2008 \text { [55] } \\
\text { USA }\end{array}$ & $\begin{array}{l}\text { I: Stepped smoking } \\
\text { cessation programme } \\
\text { (computer-assessments of } \\
\text { quit readiness; } 6 \text { weeks } \\
\text { psychological counselling, } \\
10 \text { weeks nicotine }\end{array}$ & $\begin{array}{l}322 \text { cigarette smoking } \\
\text { mental health out-patients } \\
\text { aged } 18+\text { with a diagnosis } \\
\text { of unipolar depression }\end{array}$ & RCT & $\begin{array}{l}\text { The mean costs of } \\
\text { intervention were } \$ 346 \text {. } \\
\text { Total mental health care } \\
\text { costs in the intervention } \\
\text { and control group were } \\
\$ 4805 \text { vs } \$ 4173 \text {. This }\end{array}$ & $\begin{array}{l}\text { The stepped care group } \\
\text { had } 5.5 . \% \text { greater } \\
\text { abstinence rate from } \\
\text { smoking.(p-value }<0.05 \text { ) }\end{array}$ & $\begin{array}{l}\text { Health care } \\
\text { sector }\end{array}$ & $\begin{array}{l}\text { Incremental cost per } \\
\text { successful quit } \$ 11,496 \text {. } \\
\text { Incremental cost per life year } \\
\text { gained } \$ 9,580 \text {. Cost effective } \\
74 \% \text { of time if WTP per } \\
\text { successful quit } \$ 40,000 \text {. }\end{array}$ \\
\hline
\end{tabular}

c. brief contact:

difference was not

significant.

information on quitting
and list of cessation

programmes from

counsellor.

$\begin{array}{lll}\text { Chalder et al. } & \text { I: Primary care facilitated } & 361 \text { community dwelling } \\ 2012 \text { [51] } & \text { physical activity plus usual } & \text { individuals aged 18-69 with }\end{array}$

England, UK primary care physician care first or recent new episode

Mean health and social costs per participant in the $£ 39$ greater, but this was not significant. The mean costs of the intervention for costs of the intervention for Ł252. Productivity losses were greater in the were greater in the
intervention group and this difference was almost significant at $p=0.05$ level

Craig et al. I: Integrated management 127 community dwelling 2008 [58] of mental and substance abuse disorders by

Total mean costs in the intervention and control groups were $£ 18,672$ and $£ 17,639$. This difference was disorders treated by 40 managers in community mental health teams not significant. comorbid substance use specially trained and (CMHTs).

supervised case managers

in CMHTs and 105

community dwelling

patients receiving usual case management from 39 case managers in CMHTs.

C: Standard case

18 months

The incremental cost of the exercise programme plus best care, relative to best care was $€ 2250$
No impact on substance use levels between the

Significantly greater amount Health care of physical activity at 12 group Odds Ratio 227 $p=0.0003$.

Small but non significant QALY gain of 0.014 .

up, but small positive

impact on mental health status

Body Mass Index $(p<0.003)$ Exercise: 29.7->29.4

Control: $30.6->30.8$

sector

perspective

only

2009 UK

Health care sector justice sectors

No synthesis was reported as there was no significan difference in costs or substance abuse levels gained of $£ 20,834$. $57 \%$ probability of being cost effective with WTP threshold of $£ 30,000$ per QALY gained. Not considered likely to be cost effective.

Incremental cost per QALY gained: $€ 311$. 99.9\%

probability of being cost effective if WTP of just $€ 600$ per QALY gained. 
Table 1 Economic evaluations alongside empirical studies for interventions promoting physical health for people with mental health problems (Continued)

\begin{tabular}{|c|c|c|c|c|c|c|c|}
\hline & $\begin{array}{l}\text { times per week plus simple } \\
\text { diet advice. }\end{array}$ & & & & $\begin{array}{l}\text { The mean incremental } \\
\text { Quality Adjusted Life Years }\end{array}$ & & \\
\hline & C: The standard & 6 months & CUA & & $\begin{array}{l}\text { gained was } 0.132(95 \% \\
\text { Cl:0.104-0.286) }\end{array}$ & $2005 €$ & \\
\hline & $\begin{array}{l}\text { "best primary care" : } \\
\text { routine care in general } \\
\text { practice and a } \\
\text { recommendation of } \\
\text { exercise }\end{array}$ & & & & & & \\
\hline \multirow{3}{*}{$\begin{array}{l}\text { Johnson- } \\
\text { Masotti et al. } \\
2000[48]\end{array}$} & Two interventions: & \multirow{3}{*}{$\begin{array}{l}\text { Community dwelling } \\
\text { people with severe mental } \\
\text { illness being treated on an } \\
\text { outpatient basis at risk of } \\
\text { HIV. }\end{array}$} & \multirow[t]{3}{*}{ Modelling } & \multirow{3}{*}{$\begin{array}{l}\text { The total costs of } \\
\text { intervention include staff } \\
\text { compensation, materials, } \\
\text { transportation, overhead, } \\
\text { and participants' } \\
\text { opportunity costs. Average } \\
\text { cost per person: }\end{array}$} & \multirow{3}{*}{$\begin{array}{l}\text { Infection averted per } 100 \\
\text { clients } \\
\text { Men }\end{array}$} & \multirow[t]{11}{*}{ Societal } & \multirow{11}{*}{$\begin{array}{l}\text { Advocacy training group }(A) \\
\text { was most cost effective for } \\
\text { men with incremental cost } \\
\text { per QALY gained of } \$ 48,585 \text {. } \\
\text { For women single session } \\
\text { intervention is cost saving }\end{array}$} \\
\hline & I: A multi-session small & & & & & & \\
\hline & group intervention (M) & & & & & & \\
\hline \multirow[t]{9}{*}{ USA } & \multirow{8}{*}{$\begin{array}{l}\text { I: Advocacy training (multi- } \\
\text { session that taught } \\
\text { participants to act as safer } \\
\text { sex advocates to their } \\
\text { peers).(A) }\end{array}$} & & & Single session: $\$ 178$ & S: 0.041 & & \\
\hline & & & & Multi-session: $\$ 629$ & M: 0.087 & & \\
\hline & & & & Advocacy training: $\$ 786$ & A: 0.138 & & \\
\hline & & & & & Women & & \\
\hline & & & & & S:0.098 & & \\
\hline & & & & & M: -0.041 & & \\
\hline & & & & & A: 0.019 & & \\
\hline & & & & & $\begin{array}{l}\text { QALY gains not } \\
\text { documented }\end{array}$ & & \\
\hline & $\begin{array}{l}\text { C: A single session, one-on } \\
\text {-one HIV/AIDS education } \\
\text { intervention (S) }\end{array}$ & 3 months & CUA & & & 1998 US \$ & \\
\hline $\begin{array}{l}\text { Morse et al. } \\
2006 \text { [59] }\end{array}$ & Two interventions & \multirow{3}{*}{$\begin{array}{l}149 \text { homeless people } \\
\text { treated on an outpatient } \\
\text { basis having a wide range } \\
\text { of severe mental illness with } \\
\text { substance disorder (i.e. dual } \\
\text { disorder diagnosis). }\end{array}$} & \multirow{3}{*}{ RCT } & \multirow{4}{*}{$\begin{array}{l}\text { The mean total costs for the } \\
\text { IACT }(\$ 48,764) \text { and control } \\
\text { group }(\$ 41,726) \text { were } \\
\text { significantly less than those } \\
\text { for the ACTO group }(\$ 71,211) \\
(p<0.05) \text {. }\end{array}$} & \multirow{4}{*}{$\begin{array}{l}\text { There were no differences } \\
\text { between treatment groups } \\
\text { in substance use. }\end{array}$} & \multirow[t]{3}{*}{ Health care } & \multirow{3}{*}{$\begin{array}{l}\text { Costs and outcomes were } \\
\text { separately reported. }\end{array}$} \\
\hline \multirow[t]{3}{*}{ USA } & $\begin{array}{l}\text { I: Integrated Assertive } \\
\text { Community Treatment } \\
\text { (IACT) }\end{array}$ & & & & & & \\
\hline & $\begin{array}{l}\text { I: Assertive Community } \\
\text { Treatment Only (ACTO) }\end{array}$ & & & & & & \\
\hline & C: Standard care & 24 months & CCA & & & 2001 US \$ & \\
\hline $\begin{array}{l}\text { Murphy et al } \\
2012 \text { (early } \\
\text { online) [50] }\end{array}$ & \multirow{2}{*}{$\begin{array}{l}\text { I: } 16 \text { week tailored } \\
\text { programme of exercise } \\
\text { delivered in a leisure } \\
\text { centre supervised by a } \\
\text { qualified exercise } \\
\text { professional. Plus } \\
\text { subsequent } 8 \text { month }\end{array}$} & \multirow{2}{*}{$\begin{array}{l}\text { 2,160 community dwelling } \\
\text { sedentary individuals having } \\
\text { coronary heart disease } \\
\text { (CHD) risk, and/or mental } \\
\text { health problems (mild } \\
\text { anxiety, depression/ stress } \\
\text { disorders) }\end{array}$} & \multirow[t]{2}{*}{ RCT } & \multirow{2}{*}{$\begin{array}{l}\text { Incremental cost for the } \\
\text { mental health or mental } \\
\text { health plus CHD group was } \\
£ 596 \text { but this was not } \\
\text { significant. There was a } \\
\text { small significant } \\
\text { improvement of } 0.0058 \\
\text { QALYs gained in this group. }\end{array}$} & \multirow{2}{*}{$\begin{array}{l}\text { CHD group reported } \\
\text { significantly higher levels of } \\
\text { physical activity, but no } \\
\text { difference for those referred } \\
\text { wholly or partially for } \\
\text { mental health reasons. The } \\
\text { mental health group did } \\
\text { have statistically significant }\end{array}$} & \multirow[t]{2}{*}{ Public sector } & \multirow{2}{*}{$\begin{array}{l}\text { Incremental cost per QALY } \\
\text { gained for whole population } \\
£ 12,111.89 \% \text { probability of } \\
\text { being cost effective at } \\
£ 30,000 \text { per QALY gained. } \\
£ 10,276 \text { per QALY gained for } \\
\text { mental health or mental } \\
\text { health and CHD group. }\end{array}$} \\
\hline Wales, UK & & & & & & & \\
\hline
\end{tabular}
participants to act as safer sex advocates to their peers).(A)
Life Years

nental cost

and participants'

S: 0.041

Women

s.0.098

M: -0.041

QALY gains not

documented

There were no differences between treatment groups group $(\$ 41,726)$ were in substance use.

significantly less than those

or the ACTO group $(\$ 71,211)$

have statistically significant 
Table 1 Economic evaluations alongside empirical studies for interventions promoting physical health for people with mental health problems (Continued)

\begin{tabular}{|c|c|c|c|c|c|c|c|}
\hline \multirow{5}{*}{$\begin{array}{l}\text { Pinkerton } \\
\text { et al. } 2001 \\
\text { [47] } \\
\text { USA }\end{array}$} & \multirow{2}{*}{$\begin{array}{l}\text { telephone contact by } \\
\text { exercise professional. } \\
\text { C:Usual care plus } \\
\text { information on benefits of } \\
\text { exercise and location of } \\
\text { local facilities }\end{array}$} & & & & \multirow[t]{2}{*}{$\begin{array}{l}\text { improvement in } \\
\text { depression/anxiety. }\end{array}$} & & \\
\hline & & 12 months & \multicolumn{2}{|l|}{ CUA } & & \multicolumn{2}{|l|}{2009 UKE } \\
\hline & \multirow{2}{*}{$\begin{array}{l}\text { I: Small group HIV } \\
\text { prevention programme in } \\
\text { community mental health } \\
\text { clinics, focusing on sexual } \\
\text { communication, condom } \\
\text { use skills, and motivation } \\
\text { to practice safer sex. }\end{array}$} & \multirow{2}{*}{$\begin{array}{l}87 \text { community dwelling } \\
\text { women at least being } 18 \\
\text { years old with a psychiatric } \\
\text { diagnosis of mental illness. }\end{array}$} & \multirow[t]{2}{*}{ Modelling } & \multirow{3}{*}{$\begin{array}{l}\text { Intervention cost per } \\
\text { participant: } \$ 679 \text {. Saved } \\
\$ 13,830 \text { in HIV-related } \\
\text { medical care costs. The cost } \\
\text { per } 100 \text { women was } \\
\$ 67,910 \text {, a net cost of } \\
\$ 54,080 \text { costs avoided in } \\
\text { medical care costs. For } \\
\text { sexually active women only, } \\
\text { there were } \$ 22,284 \text { in } \\
\text { avoided medical care costs } \\
\text { per } 100 \text { women. }\end{array}$} & \multirow{3}{*}{$\begin{array}{l}\text { For full sample, intervention } \\
\text { averted } 0.064 \text { infections and } \\
\text { saved } 0.40 \text { QALYs. For } \\
\text { sexually active women only, } \\
0.104 \text { infections were } \\
\text { averted and 0.64 QALYs } \\
\text { saved. }\end{array}$} & \multirow{2}{*}{ Societal } & $\begin{array}{l}\text { For full sample, cost per } \\
\text { QALY saved: } \$ 136,295 \text {. }\end{array}$ \\
\hline & & & & & & & $\begin{array}{l}\text { For sexually active women } \\
\text { only, } \$ 71,367 \text { per QALY } \\
\text { saved. }\end{array}$ \\
\hline & $\begin{array}{l}\text { C: Standard health } \\
\text { promotion programme } \\
\text { without inclusion of HIV }\end{array}$ & 6 months & CUA & & & 1999 US\$ & \\
\hline $\begin{array}{l}\text { Rosenberg } \\
\text { et al. } 2004 \\
\text { [60] }\end{array}$ & \multirow{2}{*}{$\begin{array}{l}\text { I: Specialist brief } \\
\text { programme delivered in } \\
\text { community mental health } \\
\text { centres to reduce risk of } \\
\text { blood borne infectious } \\
\text { disease. }\end{array}$} & \multirow[t]{2}{*}{$\begin{array}{l}173 \text { community dwelling } \\
\text { people with serious mental } \\
\text { illness }\end{array}$} & \multirow{2}{*}{$\begin{array}{l}\text { A 'before and } \\
\text { after' pilot study } \\
\text { at one urban } \\
\text { and one rural } \\
\text { community } \\
\text { mental health } \\
\text { centre }\end{array}$} & \multirow[t]{3}{*}{$\begin{array}{l}\text { Intervention costs per } \\
\text { person ranged between } \\
\$ 194 \text { and } \$ 262 \text {. }\end{array}$} & \multirow{3}{*}{$\begin{array}{l}\text { Increased motivation to } \\
\text { reduce risk behaviour such } \\
\text { as HIV and hepatitis } \\
\text { ( } p<0.01 \text { ). But no actual } \\
\text { decrease in self-reported } \\
\text { risk behaviour. }\end{array}$} & \multirow[t]{2}{*}{ Health care } & \multirow[t]{3}{*}{$\begin{array}{l}\text { Concluded that pilot study } \\
\text { supports feasibility and } \\
\text { efficacy of intervention. }\end{array}$} \\
\hline \multirow[t]{2}{*}{ USA } & & & & & & & \\
\hline & $\begin{array}{l}\text { C: No controls - change in } \\
\text { knowledge and risk } \\
\text { behaviours post } \\
\text { intervention }\end{array}$ & 6 months & CCA & & & 2002 US \$ & \\
\hline $\begin{array}{l}\text { Rosenberg } \\
\text { et al. } 2010 \\
\text { [61] }\end{array}$ & \multirow{2}{*}{$\begin{array}{l}\text { I: Specialist brief } \\
\text { programme delivered in } \\
\text { community mental health } \\
\text { centres to reduce risk of } \\
\text { blood borne infectious } \\
\text { disease }\end{array}$} & \multirow{2}{*}{$\begin{array}{l}236 \text { community dwelling } \\
\text { people with severe mental } \\
\text { illness and co-occurring } \\
\text { substance use disorder } \\
\text { largely from ethnic minority } \\
\text { groups. }\end{array}$} & \multirow[t]{2}{*}{ RCT } & \multirow[t]{3}{*}{$\begin{array}{l}\text { Intervention cost per } \\
\text { person: } \$ 541 \text { including } \$ 234 \\
\text { for blood tests. }\end{array}$} & \multirow{3}{*}{$\begin{array}{l}\text { People in the intervention } \\
\text { group were more likely to } \\
\text { be tested for HBV and HCV, } \\
\text { and immunised against } \\
\text { hepatitis A and hepatitis B, } \\
\text { to reduce their substance } \\
\text { abuse. However, they } \\
\text { showed no decrease in risk } \\
\text { behaviour. }\end{array}$} & \multirow{2}{*}{ Health care } & \multirow[t]{3}{*}{$\begin{array}{l}\text { Costs and outcomes were } \\
\text { separately reported. }\end{array}$} \\
\hline \multirow[t]{2}{*}{ USA } & & & & & & & \\
\hline & $\begin{array}{l}\text { C: Enhanced treatment as } \\
\text { usual. }\end{array}$ & 12 months & CCA & & & US \$ & \\
\hline $\begin{array}{l}\text { Timko et al. } \\
2006 \text { [46] }\end{array}$ & \multirow{2}{*}{$\begin{array}{l}\text { I: Community residential } \\
\text { facility acute support } \\
\text { programme for dual } \\
\text { disorder people }\end{array}$} & \multirow{2}{*}{$\begin{array}{l}57 \text { community dwelling and } \\
173 \text { hospital with dual } \\
\text { psychiatric disorder and } \\
\text { substance abuse diagnosis }\end{array}$} & \multirow[t]{2}{*}{ RCT } & \multirow{2}{*}{$\begin{array}{l}\text { Mean health care costs for } \\
\text { the community group were } \\
\$ 21,966 \text { compared with } \\
\$ 33,188 \text { in the hospital } \\
\text { group. This difference was } \\
\text { not significant. }\end{array}$} & \multirow{3}{*}{$\begin{array}{l}\text { The community group had } \\
\text { significantly improved } \\
\text { Addiction Severity Index } \\
\text { Scores compared to the } \\
\text { hospital group. } 26 \% \text { of the } \\
\text { community group were in } \\
\text { remission compared with } \\
16 \% \text { of the hospital group. } \\
\text { This was not significant }\end{array}$} & \multirow[t]{2}{*}{ Health care } & \multirow{3}{*}{$\begin{array}{l}\text { Costs and outcomes } \\
\text { reported separately, but } \\
\text { noted that mean costs for } \\
\text { patients in remission in } \\
\text { community group of } \\
\$ 12,174 \text { were less than half } \\
\text { those of hospital group. }\end{array}$} \\
\hline \multirow[t]{2}{*}{ USA } & & & & & & & \\
\hline & $\begin{array}{l}\text { C: Hospital inpatient acute } \\
\text { support programme for } \\
\text { dual disorder people }\end{array}$ & 12 months & CCA & $\begin{array}{l}\text { For those patients } \\
\text { successfully in remission } \\
\text { from substance abuse cost }\end{array}$ & & 2003 US\$ & \\
\hline
\end{tabular}




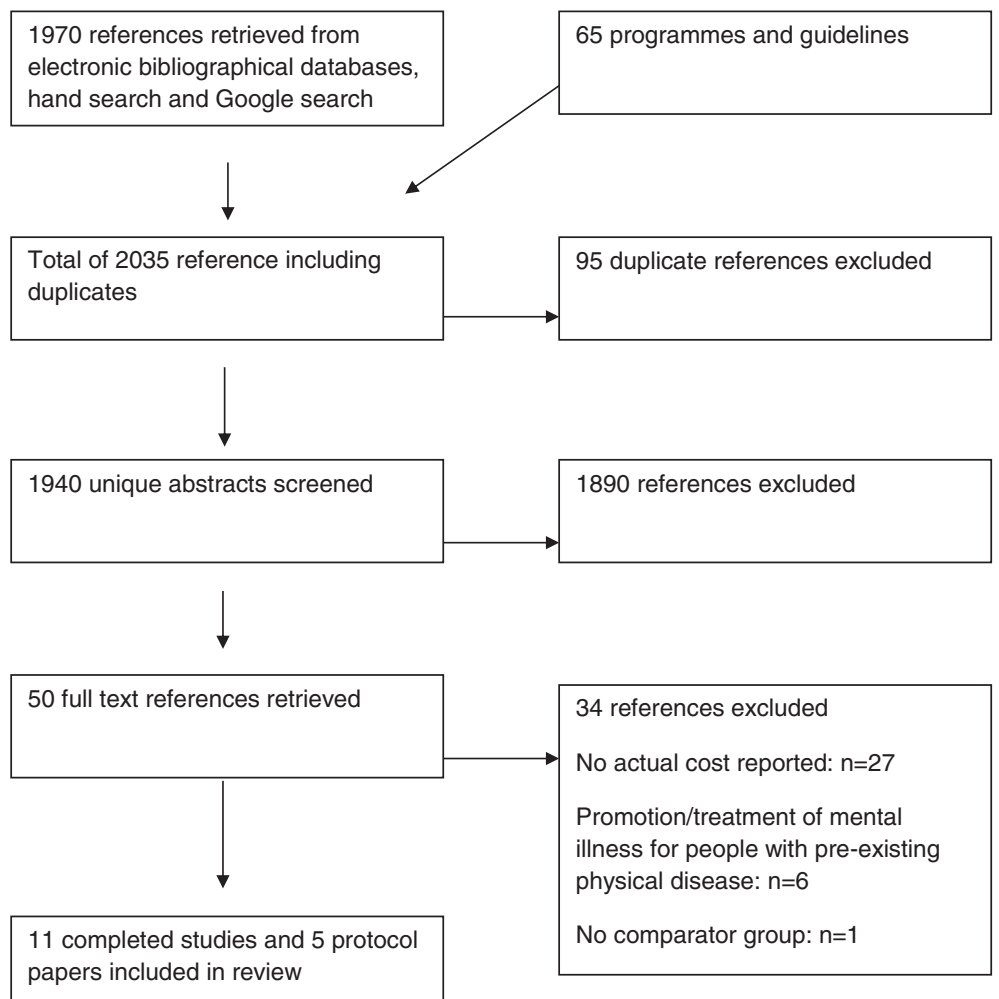

Figure 1 Literature search flow chart.

QALY threshold of just $\$ 865$ ( $€ 6002003$ prices). These QALY gains may be conservative if the increased level of physical activity can be maintained over the longer term.

In Wales the National Exercise Referral Scheme has also been subject to economic evaluation [50]. Sedentary individuals aged 17 or older with coronary heart disease risk factors and/or mild anxiety, depression or stress were randomised to receive a 16 week tailored exercise programme delivered by exercise professionals using motivational interview techniques to encourage the long term sustainability of behaviour change. During this time and for the subsequent eight months they were followed up by phone by the exercise professional to help reduce the risk of relapse. The intervention was found to significantly increase physical activity in those with coronary risk only; it did not have an impact on physical activity in those with mental health problems, although there were significant reductions in their depression or anxiety status, suggesting that they may have benefited from social contact arising from participation in the scheme. The cost per QALY gained for the mental health group was $\$ 15,808$ (£10,276, 2009 prices) compared with
$\$ 18,631$ (£12,111, 2009 prices) for the whole mental health and coronary heart disease risk group. There was an $89 \%$ of being cost effective for the whole study population with a cost per QALY threshold of $\$ 46,150$ (£30,000, 2009 prices).

A smaller scale randomised controlled trial looking at professionally facilitated physical activity for people with depression compared to usual primary care was undertaken in England [51]. While the central aim was to look at the impact of exercise on depression, for which no impact was found, the study reported that the rate of sustained physical activity in the intervention group was more than double that of the control group at 12 month follow up. This finding is important given the challenges in motivating people with depression to engage in physical activity. Therefore while the intervention was not considered to be cost effective as an intervention to tackle depression, with little impact on recorded QALY outcomes during the lifetime of the study, the intervention may have potential in the longer term to be cost effective, if the increase in physical activity in this population group can be sustained and further benefits to physical health realised. 
Table 2 Protocol papers for current economic evaluations

\begin{tabular}{|c|c|c|c|c|c|c|c|}
\hline $\begin{array}{l}\text { Bibliographic } \\
\text { information }\end{array}$ & $\begin{array}{l}\text { Intervention (I) } \\
\text { Comparator (C) }\end{array}$ & Target Population /Duration & $\begin{array}{l}\text { Study Design } \\
\text { Type of analysis }\end{array}$ & Cost results & $\begin{array}{l}\text { Physical health } \\
\text { related } \\
\text { Effectiveness } \\
\text { results }\end{array}$ & $\begin{array}{l}\text { Perspective } \\
\text { /Price year }\end{array}$ & $\begin{array}{l}\text { Synthesis } \\
\text { of costs } \\
\text { and effects }\end{array}$ \\
\hline $\begin{array}{l}\text { Bonevski et al. } \\
2011[57]\end{array}$ & \multirow{2}{*}{$\begin{array}{l}\text { I: Client centred, caseworker-delivered } \\
\text { cessation support intervention for a } \\
\text { socially disadvantaged population. }\end{array}$} & \multirow{2}{*}{$\begin{array}{l}200 \text { community dwelling socially } \\
\text { disadvantaged smokers including } \\
\text { people with mental health } \\
\text { problems attending a community } \\
\text { social service centre }\end{array}$} & \multirow[t]{2}{*}{ RCT } & \multirow{3}{*}{$\begin{array}{l}\text { Resources and costs of } \\
\text { interventions and impacts } \\
\text { on health service use }\end{array}$} & \multirow[t]{3}{*}{$\begin{array}{l}\text { Changes in smoking } \\
\text { behaviour }\end{array}$} & \multirow[t]{3}{*}{$\begin{array}{l}\text { Health } \\
\text { system only }\end{array}$} & \multirow[t]{3}{*}{ Not stated } \\
\hline \multirow[t]{2}{*}{ Australia } & & & & & & & \\
\hline & $\begin{array}{l}\text { C: Information on smoking cessation and } \\
\text { telephone number for Quitline }\end{array}$ & 12 months & CCA & & & & \\
\hline $\begin{array}{l}\text { Carter et al. } \\
2012 \text { [52] }\end{array}$ & \multirow{3}{*}{$\begin{array}{l}\text { I: } 12 \text { week preferred intensity aerobic } \\
\text { exercise, with motivational coaching and } \\
\text { support }\end{array}$} & \multirow{3}{*}{$\begin{array}{l}\text { Community dwelling people } \\
\text { aged } 14-17 \text { already in contact } \\
\text { with health care services with } \\
\text { depression }\end{array}$} & \multirow[t]{3}{*}{$\begin{array}{l}\text { RCT plus focus } \\
\text { group analysis }\end{array}$} & \multirow{4}{*}{$\begin{array}{l}\text { Boundaries not stated but } \\
\text { will use Client Service } \\
\text { Receipt Inventory to record } \\
\text { health care service use. }\end{array}$} & $\begin{array}{l}\text { Changes in } \\
\text { depression using }\end{array}$ & \multirow[t]{4}{*}{ Not stated } & \multirow{4}{*}{$\begin{array}{l}\text { Incremental } \\
\text { cost per } \\
\text { QALY } \\
\text { gained }\end{array}$} \\
\hline \multirow[t]{3}{*}{ England, UK } & & & & & $\begin{array}{l}\text { Children's } \\
\text { Depression Inventory }\end{array}$ & & \\
\hline & & & & & QALYs using EQ-5D & & \\
\hline & C: Usual care & 9 months & CUA, CEA & & $\begin{array}{l}\text { Physical Activity } \\
\text { Intensity (Borg Scale) }\end{array}$ & & \\
\hline $\begin{array}{l}\text { Kruisdijk et al. } \\
2012 \text { [54] }\end{array}$ & $\begin{array}{l}\text { I: } 6 \text { months exercise therapy or Nordic } \\
\text { walking plus usual care }\end{array}$ & $\begin{array}{l}\text { People aged } 18-65 \text { with diagnosis } \\
\text { of depression or bipolar disorder } \\
\text { who are being treated on an } \\
\text { inpatient or outpatient basis }\end{array}$ & $\mathrm{RCT}$ & $\begin{array}{l}\text { Resource use and costs } \\
\text { determined using Trimbos/ } \\
\text { iMTA Questionnaire for } \\
\text { Costs associated with }\end{array}$ & $\begin{array}{l}\text { Risk factors for } \\
\text { metabolic syndrome. } \\
\text { Fitness and Physical } \\
\text { Activity. QALYs using }\end{array}$ & $\begin{array}{l}\text { Health care } \\
\text { use and } \\
\text { productivity } \\
\text { losses }\end{array}$ & $\begin{array}{l}\text { Incremental } \\
\text { cost per } \\
\text { QALY } \\
\text { gained }\end{array}$ \\
\hline $\begin{array}{l}\text { The } \\
\text { Netherlands }\end{array}$ & C: Usual care & 12 months & CUA & Psychiatric IIIness (TIC-P) & EQ-5D & & \\
\hline $\begin{array}{l}\text { Stockings et al. } \\
2011[56]\end{array}$ & \multirow{2}{*}{$\begin{array}{l}\text { I: Multi-modal smoking cessation } \\
\text { intervention (brief motivational } \\
\text { interviewing plus range of post discharge } \\
\text { support for up to } 16 \text { weeks. }\end{array}$} & \multirow{2}{*}{$\begin{array}{l}200 \text { smokers aged } 18+\text { in an } \\
\text { inpatient facility with acute } \\
\text { mental health problems }\end{array}$} & \multirow[t]{2}{*}{$\mathrm{RCT}$} & \multirow{3}{*}{$\begin{array}{l}\text { Resource use and costs } \\
\text { needed to deliver } \\
\text { intervention }\end{array}$} & \multirow{3}{*}{$\begin{array}{l}\text { Changes in smoking } \\
\text { behaviour. Use of } \\
\text { alcohol and other } \\
\text { substance abuse. } \\
\text { Mental wellbeing }\end{array}$} & \multirow[t]{3}{*}{$\begin{array}{l}\text { Health } \\
\text { system only }\end{array}$} & \multirow[t]{3}{*}{ Not stated } \\
\hline \multirow[t]{2}{*}{ Australia } & & & & & & & \\
\hline & $\begin{array}{l}\text { C: Hospital smoking care only includes a } \\
\text { referral to Quitline on discharge. }\end{array}$ & 6 months post discharge & CCA & & & & \\
\hline $\begin{array}{l}\text { Verhaeghe } \\
\text { et al. } 2012 \text { [53] }\end{array}$ & \multirow{2}{*}{$\begin{array}{l}\text { I: } 10 \text { week health promotion intervention } \\
\text { (psycho-educational and behavioural } \\
\text { group sessions, supervised exercise, } \\
\text { individual counselling) targeting physical } \\
\text { activity and diet plus usual care }\end{array}$} & \multirow{2}{*}{$\begin{array}{l}\text { People aged } 18 \text { - } 75 \text { with mental } \\
\text { disorders living in sheltered } \\
\text { housing. } 201 \text { in intervention; } 83 \\
\text { in control group } 9 \text { months (RCT) }\end{array}$} & \multirow{2}{*}{$\begin{array}{l}\text { Cluster RCT and } \\
\text { Markov Modelling } \\
\text { to extrapolate risk } \\
\text { of CVD and } \\
\text { diabetes over } 10 \\
\text { years }\end{array}$} & \multirow{3}{*}{$\begin{array}{l}\text { Intervention costs plus } \\
\text { costs of health care } \\
\text { utilisation }\end{array}$} & \multirow{3}{*}{$\begin{array}{l}\text { BMI, Waist } \\
\text { Circumference, body } \\
\text { weight, fat mass, } \\
\text { QALYs using SF-36 }\end{array}$} & \multirow[t]{3}{*}{$\begin{array}{l}\text { Health } \\
\text { system }\end{array}$} & $\begin{array}{l}\text { Incremental } \\
\text { cost per }\end{array}$ \\
\hline Belgium & & & & & & & $\begin{array}{l}\text { QALY } \\
\text { gained }\end{array}$ \\
\hline & C:Usual care & 10 years (model) & CUA & & & & \\
\hline
\end{tabular}


The number of lifestyle behaviour modification evaluations will grow as we also identified three protocol papers. In England a published protocol describes an economic evaluation alongside a trial of self-determined intensity one hour per week for 12 weeks aerobic exercise programme for community dwelling people aged 14-17 with depression [52]. Two of the protocols will consider people either in residential or inpatient settings. One is for a cost utility analysis alongside a cluster randomised controlled trial comparing the use of a ten week health promotion programme versus usual care for people with a range of mental health problems living in sheltered housing in Flanders, Belgium [53]. The programme, which is being delivered to more than 200 people, includes group-based educational sessions, supervised exercise and individual counselling. While empirical data will only cover a six month follow up period, modelling will be used to estimate the impacts of sustained behaviour change on risks of cardiovascular disease and diabetes over a ten year period.

The second study in the Netherlands is for a controlled trial on the use of regular hour=long running therapy or Nordic walking programmes over six months by people of working age with depression or bipolar disorder being treated on an inpatient or outpatient basis [54]. Impacts on metabolic risk factors, physical activity rates and mental health will be assessed over one year. All three of these studies will measure several outcomes including incremental costs per QALY gained.

\section{Programmes to reduce smoking, alcohol and substance abuse}

Another key concern is to tackle addictive behaviours such as smoking, alcohol and illicit substance abuse in people with mental health problems. However we were only able to identify one cost effectiveness study looking specifically at smoking cessation interventions for people with mental health problems. This study compared a stepped cessation programme (three computer-mediated assessments of readiness to quit smoking, six psychological counselling sessions, up to ten weeks nicotine replacement therapy, and offer of sustained-release bupropion and two additional counselling sessions) in outpatient care settings with brief care for smokers with depression [55]. After 18 months the stepped care group had a $5.5 \%$ greater chance of ceasing smoking $(\mathrm{P}<0.05)$ at a cost per quit of $\$ 13,519(\$ 11,496$, 2003 prices) or $\$ 11,266$ (\$9,580, 2003 prices) per life year gained. In sensitivity analysis, applying a cost effectiveness threshold of $\$ 40,000$ per successful quit, there was a $74 \%$ chance that the smoking cessation programme would be cost effective.

We also identified two study protocols from Australia related to smoking cessation interventions. One is looking at the integration of multi-modal smoking cessation strategies at an Australian inpatient facility and postdischarge, including an analysis of cost impact [56]. The second will evaluate the effectiveness and cost consequences of smoking cessation advice provided to socially vulnerable smokers, including those with mental health problems, at a community social services centre [57].

We also identified studies looking at the economic case for tackling the harmful effects on health of substance use in individuals with a dual mental disorder / substance abuse diagnosis. In south London, one evaluation looked at the costs and effectiveness of an integrated care programme using case managers trained to deal with substance use disorders in people with severe mental illness [58]. After 18 months, no significant differences in costs, alcohol consumption or cannabis use were observed between the integrated care group and waiting list controls. A lack of continuity in case management due to high staff turnover, diverting resources to staff retraining and retention mechanisms may have hampered the intervention, while the duration of the study was also felt by the authors not to be long enough to achieve significant changes in substance use.

In the USA, a small study with 149 participants also explored costs and outcomes of integrated treatment for both mental health needs and substance abuse by the health care team plus Assertive Community Treatment (IACT), compared with Assertive Community Treatment Only (ACTO) or standard care (SC) for homeless people with a dual diagnosis [59]. While the study reported no significant difference in substance abuse or mental health outcomes, the IACT and ACTO groups were significantly more likely to be in stable housing $(\mathrm{P}=0.03)$; they were also significantly more likely to be satisfied with their care $(\mathrm{P}=0.03)$. Over 24 months, IACT and SC had significantly lower costs than ACT (p value not reported). The ACTO group's costs may have been higher because they did not follow their own protocol and made much use of external outpatient mental health services rather than using their own team psychiatrist.

Another randomised controlled trial from the US evaluated costs and outcomes for 230 people with a dual diagnosis of co-morbid substance use and psychiatric disorders initially assigned to either a hospital or community residential facility (CRFs) acute care programme [46]. Over one year, patients in the CRF group had Addition Severity Index (ASI) scores that were significantly lower for both alcohol use $(\mathrm{p}<0.01)$ and drug use $(\mathrm{P}<0.05)$ compared to the hospital group. Patients with moderate psychiatric disorder severity showed better ASI scores for alcohol use in CRFs $(\mathrm{p}<0.05)$, however patients with the highest levels of severity had better outcomes in the hospital group, although this difference was not statistically significant. While there was no statistically significant difference in overall health care costs between the groups, 
for those patients successfully in remission, health care costs in the CRF group were \$14,317, (\$12,174, 2003 prices) which was just over half the costs of similar patients in the hospital care group. While acknowledging that these results should be interpreted with caution due to small sample size, the authors suggested that shifting more patients with less severe disorders away from hospital care to community-based care could potentially lead to cost-savings, especially if in addition to a reduction in substance abuse other beneficial aspects of community care in terms of its less restrictive nature and promotion of social inclusion, were also considered.

\section{Programmes to reduce the risk of infectious disease}

People with dual diagnosis in particular are more likely to be exposed to infectious disease such as HIV/AIDS, hepatitis B and hepatitis $C$. Four economic studies of interventions to reduce the risk of infectious disease were also identified. Some were targeted at high risk populations such as people with both severe psychiatric disorders and substance abuse.

The costs and outcomes of the brief STIRR (Screen, Test, Immunise, Reduce risk, and Refer) intervention delivered by a mobile specialist team to prevent or detect and treat blood-borne diseases in people with serious mental health problems have been evaluated. Initially an uncontrolled pilot study in two community mental health centres in New Hampshire [60] reported significant improvements in the levels of knowledge about blood-borne infectious diseases $(\mathrm{p}<0.01)$ and in motivation for prevention before and after the intervention $(\mathrm{p}<0.01)$, but no decrease was found in self-reported risk behaviours. The cost of the programme ranged from $\$ 233$ to $\$ 315$ (\$194 to $\$ 262$, 2002 prices) per participant. Subsequently the costs and outcomes of the programme were assessed in a randomised controlled trial delivered to a dual diagnosis urban population [61]. Compared with enhanced treatment as usual, there were modest significant positive changes in the DALI (Dartmouth Assessment of Lifestyle Instrument) scores for alcohol use $(\mathrm{p}<0.022)$. Moreover, the STIRR group showed significant improvements in clinician-rated Drug Use Scale scores $(\mathrm{p}<0.034)$. However, there were no significant changes in HIV knowledge or in risky behaviours. Programme costs per participant were \$551 (\$541, 2008 prices) per participant (2008 US\$). However, the costs of care as usual, as well as any consequent impacts related to health care resource utilisation were not reported.

In the US, Johnson-Masotti et al. performed a costutility analysis of three cognitive-behavioural HIV/AIDS risk reduction interventions for people with severe mental illness, synthesising data using mathematical modelling for a three-month period [48]. The interventions were a single session (one-on-one) HIV education intervention, a multiple-session small group intervention, and a multisession group intervention for teaching peer advocacy skills. Gender differences were observed in interventions that were most cost effective, with advocacy training for men having an incremental cost per QALY gained compared to a single session intervention of $\$ 62,875$ ( $\$ 48,585$, 1998 prices) a figure that would be considered cost effective in a US context. For women, while the single session intervention was actually cost saving compared to doing nothing, the multi-session intervention was less effective at higher cost, while investing in advocacy training would not have been cost-effective at an incremental cost of $\$ 603,062$ (\$465,994, 1998 prices) per QALY gained.

Another US study looked at the cost-effectiveness of a nine session small-group intervention to prevent HIVinfection in women with mental disorders [47]. It suggested that a targeted approach may be more cost effective than a population wide strategy. For the whole study population a cost per QALY gained of $\$ 173,828$ ( $\$ 136,295$, 1999 prices) was seen, compared with a cost per QALY of $\$ 91,020$ (\$71,367, 1999 prices) for high risk women, who had been sexually active in the previous three months. Although still well above the often suggested threshold value of $\$ 50,000$ per QALY gained in the USA [62], there may have been potential for an even more targeted strategy to be cost effective.

\section{Discussion}

There is a growing body of clinical effectiveness studies on programmes to prevent somatic diseases in people with mental disorders [39-42,63,64]. We have also indicated that the importance of protecting physical health is to be found in different mental health policy plans. While there is an evidence base on the cost effectiveness of many health promoting interventions for the general population, our review indicates that there is still very little information available on the cost-effectiveness of these interventions for people with pre-existing mental health problems.

One previous review found no cost-effectiveness studies of lifestyle interventions on the physical activity and eating habits of people with severe mental disorders [41], while a recent Cochrane review was unavailable to find a single randomised controlled trial reporting evidence on the effectiveness or cost effectiveness of guidelines advising on the monitoring of physical health in people with severe mental health problems [65]. Similarly a review of programmes to promote the physical health of people with mental health problems was only able to find some brief information on the commercial costs of obtaining manualised programmes rather than any evidence of their cost effectiveness [39].

Smoking cessation has also been highlighted as a key goal for the protection of physical health in people with mental health problems, given the higher rates of smoking 
in these populations compared with the general population [66]. While there is an extensive literature on the cost effectiveness of different smoking cessation strategies for the general public, indicating that highly cost effective smoking cessation interventions are available $[67,68]$, we were only able to identify one economic evaluation of a smoking cessation study targeted at people with mental health needs [55].

We were also only able to identify three economic evaluations of interventions to encourage physical activity, all of which focused on depression and or anxiety disorders rather than also being targeted at people with other severe mental disorders. There is a need to have more studies focusing also on addressing physical health promotion in individuals with severe mental illness, particularly given their higher risks of metabolic syndrome as a result of medication use.

This situation may however be changing; two physical activity related studies in our review were published in 2012 and there are three study protocols looking at the cost effectiveness of healthy lifestyle promoting interventions, two of which will target people with more severe mental illness who are being treated in inpatient and residential settings. A further two study protocols we identified will look at smoking cessation interventions, including one for residents of an acute psychiatric inpatient care facility.

Overall, most of the studies we identified suggest that there is an economic case for protecting the physical health of people with mental health needs, and most report incremental cost effectiveness ratios such as cost per smoking quit achieved or cost per QALY gained.

\section{Strengthening the literature}

There remain substantial limitations and research gaps with the existing literature. Here we briefly discuss issues around uptake and behaviour change; population subgroup analysis; the timeframe of studies; study perspective; sample size; transferability to different contexts; better understanding of issues of fidelity in implementation; and the potential for economic modelling.

\section{Uptake and behaviour change}

Firstly, there was little exploration in this analysis of factors that either inhibit or promote sustained behaviour change. This is of particular importance when considering health promoting interventions which require regular behaviour change and/or participation in an activity over a period of time to have an impact, e.g. exercise and diet programmes. Economic analyses need to consider the extent to which these uptake issues apply to interventions to address physical co-morbidity in this population and what additional costs are incurred as a result of effective measures taken to promote better uptake [69]. It may well be the case that interventions that currently do not appear cost effective may be seen in a more favourable light if resources were invested in better tailoring of programmes and interventions to people with mental health needs.

This also raises the possibility of looking at the cost effectiveness of techniques from behavioural psychology or economics to influence individual behaviour patterns and perhaps 'nudge' people towards different types of behaviours. Recent reviews of this literature in respect of people with mental health problems are tentatively positive $[70,71]$, requiring further research not only on effectiveness but also on the costs of implementation.

In this context nurses and other health care professionals such as physical health and lifestyle trainers that routinely work closely with people with mental health problems can focus more on looking at factors that may encourage greater sustained participation in health promoting activities [72]. Strengthening the training of professionals in somatic care, as well as going beyond the absence of mental disorder to consider the benefits of positive wellbeing, can only be helpful for lifestyle monitoring and behavioural change [73]. Better training will also reduce the likelihood that these professionals 'avoid' dealing with physical health issues through a lack of expertise.

\section{Sub-group analyses}

It is also important to consider cost effectiveness for population sub-groups as cost effectiveness ratios for the population as a whole may not reflect the cost effectiveness of the intervention for specific population groups, for instance related to age, gender, diagnosis or living conditions [74]. Few of the studies undertaken to date have looked at sub-groups, although there can be very different conclusions drawn on what is cost effective, as in the case of the evaluation of HIV risk reduction programmes for men or women [48]. It may also be the case, for example, that it might be easier to encourage individuals to take up physical activities when they are living in residential accommodation which includes a gym or other sporting equipment, compared with individuals who are living independently in the community and may not have easy access to this equipment. In the same way it may be more challenging for individuals to eat healthily if they are living in areas with little easy access to fresh fruit and vegetables, but many fast food restaurants [75]. This is also important when considering the implications for health inequalities of any intervention. Health promoting interventions for the general population can in some circumstances widen health inequalities within the population because of different rates of uptake and capacity to benefit [76].

\section{Study timeframes}

We also know comparatively little about the long term effectiveness and therefore long term cost effectiveness of 
interventions. Most studies had a short-term follow-up period between three and 12 months. Only three studies covered longer periods from 18 to 24 months. We have noted that health promotion interventions may require regular long term use to ensure sustained behaviour change, let alone have a sustained impact on physical health. In a shorter time frame, studies may have to rely on intermediate outcomes such as abstinence from smoking or harmful drinking or changes in dietary behaviour. These intermediate indicators may not always immediately translate into anticipated health gains, although there may be short term benefits to be flagged up e.g. from social capital gained through socialisation and networking with other participants in an exercise class. Economic analysis should also consider the economic benefits of any improvements in mental health arising from health promoting interventions.

\section{Study perspective}

It is also important to consider the impact of study perspective on the cost effectiveness. Some of the costs of health promoting interventions might be borne outside the health care system, e.g. local government may be responsible for some aspects of sports and recreation activities, while there will also be external economic benefits such a potential reduction in the need for informal care support from family members and a reduction in time spent out of work for people of working age. Some of these broader impacts may make the case for investment stronger. Better physical health may remove one barrier to maintaining or gaining a job, improving self-care and self-management skills, as well as improving quality of life and sense of social inclusion in the local community. However most studies we identified focused solely on the perspective of the health care budget holder alone and did not consider these broader impacts. Only four studies looked at costs from a broader viewpoint, including costs to the criminal justice system [58], the public purse [51] and the opportunity costs incurred by study participants $[47,48]$.

\section{Sample size}

Another challenge is the lack of statistically significant differences in costs between interventions and comparators in a number of studies. This is by no means unique to this literature, but it means that studies may have been powered to detect significant differences in effect size between interventions and control groups, but not any significant differences in cost $[46,48,49,59,61]$. In studies with small sample sizes, skewed costs driven by a few unusually costly individuals in one community can also distort potential average cost per participant $[59,77]$.

\section{Transferability across contexts}

Most studies are concentrated in the US; but regardless of where a study is set how generalisable are findings to other contexts? Uptake and use of interventions may be influenced by the organisation, structure and culture of health care systems in different countries. Even within a country, especially where there are significant differences in structures between regions, e.g. between American states, cost effectiveness findings on programmes may not be easily generalisable [59]. In the case of the Spanish walking for health programme, the high rate of recruitment $(79 \%)$ had an influence on programme cost effectiveness. A low cost intervention, a letter from a general practitioner sent to women in the target population, was sufficient to encourage participation in the programme [49]. In countries where primary care does not play such a pivotal role, different, potentially more expensive, methods of engaging with the target population may be required.

It is also important to explicitly report resources used separately from their costs in order to aid future adaptation of study results [69] [78] and to be aware of the fidelity in the way that programmes have been implemented in different contexts; a lack of fidelity in complying with recommended practice protocols meant that Integrated Assertive Community Treatment was reported to be less costly than standard Assertive Community Treatment in one study in our analysis [59].

\section{Fidelity in implementation}

Process and context evaluation alongside economic analyses is also crucial to monitor the fidelity in implementation of interventions. Consultation with experts can be used to get a sense of the feasibility of delivering an intervention to a minority population group, for example. In the STIRR programme, cultural competence experts/ethnographers, who were specialised in urban, AfricanAmerican culture checked any potential cultural issues with pilot participants from ethnic minority backgrounds before delivering the intervention to the larger study population [61]. Discussions with local experts can also help to identify any potential differences and resource requirements that are likely to be observed when adapting an intervention to a different context.

\section{Using models}

Economic models can be used to synthesise data on costs and benefits; crucially they can be used to project long term costs and benefits well beyond the duration of any empirical study. Yet they appear to have been rarely used for interventions for this client group thus far, although one of the study protocols identified in this review will model ten year impacts on cardiovascular disease and diabetes of a health promotion intervention [53]. 
Cost and resource parameters and potential health promotion pathways and outcomes can also be adapted in models to take account of different infrastructure contexts, cultural factors or different levels of engagement in different population groups. Different scenarios can be constructed to take account of different probabilities of uptake and participation in population sub-groups, for instance accounting for differences in the severity of symptoms for different mental disorders or accommodation status. Impacts on quality of life and other outcomes can also be varied in modelling analyses. The potential long term benefits to health of sustained change in health promoting behaviours can also be projected in models.

Sensitivity analysis with these models can be used to explore the minimum level of effectiveness that would be required for an intervention to be cost effective in different country contexts. This in turn can help decision makers determine whether piloting a health promoting approach developed elsewhere is feasible. Such analyses might also help in better targeting health promoting interventions, or in adapting them to the needs of different population sub-groups.

\section{Limitations of the review}

Although our review was not restricted to English language materials, no studies in other languages were identified. One potential bias may be that we did not search any non-English language bibliographic databases, nor did we search for terms in languages other than English. We also excluded studies such as exercise programmes for people with mental health problems which at first sight intuitively appear to be relevant. These were excluded because they did not report physical health outcomes, however, much may be learnt on the acceptability of exercise programmes from these studies. In addition, quite a few economic evaluation studies excluded were for interventions to prevent and treat infectious diseases such as HIV/AIDS, hepatitis B and hepatitis C for people with substance abuse without any official diagnosis of mental illness. Given the high level of co-morbidity between mental illness and substance abuse, it may be the case that much can be learnt from these studies.

We also did not look at the cost effectiveness of any pharmacological developments that may reduce any risk of adverse events such as weight gain and metabolic syndrome, from antipsychotics or antidepressants. Although excluded from our analysis, we did identify one such study [79]. This is an area that also merits further future analyses, including exploration of combined health promotion/pharmacological interventions. It may also be the case that useful data from specialist early intervention teams for mental health may have been overlooked, given that these teams often include physical health specialists.
To date however, economic studies of these teams have focused on impacts on mental health alone [80].

The small number of studies identified, coupled with limited diagnostic differentiation, also means that we have to be very cautious in our interpretation of the findings of this review. Interventions looking at physical activity and smoking cessation have been targeted at people with depression and anxiety disorders; the merits of these interventions in promoting the physical health of people with other severe mental illness need to be assessed. Equally, actions to prevent substance abuse and infectious disease have concentrated on severe mental illness and need to be explored with other population groups.

Despite these limitations we believe we have conducted the most comprehensive review to date of economic evaluations for physical health promotion in people with mental health problems. Economic evaluations covering interventions to promote physical activity and better dietary behaviour, discourage smoking, substance and alcohol consumption and reduce the risk of blood borne disease specifically in populations with mental health needs, both within inpatient facilities and in the community, have been identified.

\section{Conclusions}

There is a very small, albeit growing, literature on the cost effectiveness of interventions to promote the physical health of people with mental health problems. Most of these studies suggest that value for money actions in specific contexts and settings are available. What is clear however is that as the success or failure of health promoting interventions can be very context specific, more studies are needed in more settings, reporting outcomes in a common metric, such as quality of life years gained, and showing resource use and costs in a transparent manner, including costs beyond the health care system.

In the short-term, economic modelling techniques might be better utilised to explore possible costs and benefits under different scenarios and over different time horizons, making use of a wide range of sensitivity analyses to account for uncertainty in data parameters. Such models can synthesise data from existing effectiveness reviews $[40,41]$. In the mid to longer term it would be helpful to at least collect data on resource use and cost impacts alongside effectiveness data in new trials. This can facilitate better comparability across different interventions and ultimately help policymakers in the public health arena to prioritise resource allocations to specific preventive strategies.

Finally it should never be forgotten that economic evaluations are not conducted in isolation. Apart from costeffectiveness, it is crucial to look at barriers and facilitators to intervention by being mindful of the findings of any process evaluation, so as to take into account issues such 
as programme fidelity, uptake and adherence. The role of behavioural psychological techniques to influence health behaviours and mitigate any adverse impact on health inequalities might also be considered. Looking at the success or failure of the organisation of any system to promote physical health can also be the subject of economic analysis, for instance in terms of different types of multidisciplinary team work, links between primary and specialist care services, and in the co-ordination and continuity of mental and physical health care services.

\section{Additional files}

\section{Additional file 1: PRISMA Checklist.}

Additional file 2: PubMed/Medline search strategy.

\section{Abbreviations}

ACTO: Assertive community treatment only; AIDS: Acquired immune deficiency syndrome; ASI: Addition severity index; BMI: Body mass index; CBA: Cost benefit analysis; CCA: Cost consequences analysis; CEA: Cost effectiveness analysis; CHD: Coronary heart disease; COA: Cost offset analysis; CRFs: Community residential facilities; CUA: Cost utility analysis; DALI: Dartmouth assessment of life instrument; DALY: Disability adjusted life years; EQ-5D: EuroQOL; HIV: Human immunodeficiency virus; IACT: Integrated assertive community treatment; QALY: Quality adjusted life years; RCT: Randomised Controlled Trial; SF-36: Shortform (36) health survey; STIRR: Screen, test, immune, reduce risk, and refer; UK: United Kingdom; US: United States; WTP: Willingness to pay.

\section{Competing interests}

DM is an associate editor of BMC Public Health. The authors declare that they have no other conflicts of interests.

\section{Authors' contributions}

ALP, DM, TB and RK conceived the manuscript. DM and ALP were mainly responsible for search strategy design, study selection and analysis and drafting the manuscript. PW, CVG, TB and RK separately searched for evidence on the effectiveness of interventions. ALP and DM wrote the first draft of the manuscript. PW, CVG, TB and RK contributed to the writing of the manuscript. All authors agree with the manuscript results and conclusions. All authors read and approved the final manuscript.

\section{Acknowledgements}

This review was undertaken as part of the European network for promoting the physical health of residents in psychiatric and social care facilities (HELPS project), which was funded by the European Union in the framework of the Public Health Programme (Contract Number: 2006334).

\section{Author details \\ ${ }^{1}$ Personal Social Services Research Unit, London School of Economics and Political Science, London, UK. ${ }^{2}$ European Observatory on Health Systems and Policies, London School of Economics and Political Science, London, UK ${ }^{3}$ Department of Psychosomatic Medicine, Psychotherapy/Psychiatry, University of Mainz, Mainz, Germany. ${ }^{4}$ Department of Psychiatry II, Ulm University, Bezirkskrankenhaus Günzburg, Germany.}

Received: 9 January 2013 Accepted: 16 August 2013

Published: 29 August 2013

\section{References}

1. Government HM: No health without mental health. A cross-government mental health outcomes strategy for people of all ages. London: Department of Health; 2011.

2. Government S: Mental health strategy for Scotland: 2012-2015. Edinburgh: Scottish Government; 2012

3. Ministère chargé de la Santé: Plan psychiatrie et Santé mentale 2011-2015. Paris: Mlnistere des Affaires sociales et de la Santé; 2011.
4. Coghlan R, Lawrence D, Holman CDJ, Jablensky AV: Duty to care: physical illness in people with mental illness. Perth: University of Western Australia; 2001.

5. Gough SCL: Diabetes and schizophrenia. Practice Diabetes International 2005, 22:23-26.

6. De Hert M, Correll CU, Bobes J, Cetkovich-Bakmas M, Cohen D, Asai I, Detraux J, Gautam S, Moller HJ, Ndetei DM, et al: Physical illness in patients with severe mental disorders. I. Prevalence, impact of medications and disparities in health care. World Psychiatry 2011, 10(1):52-77.

7. Kilian R, Becker T, Kruger K, Schmid S, Frasch K: Health behavior in psychiatric in-patients compared with a German general population sample. Acta Psychiatr Scand 2006, 114(4):242-248.

8. Harris EC, Barraclough B: Excess mortality of mental disorder. Br J Psychiatry 1998, 173:11-53

9. Fleischhacker WW, Cetkovich-Bakmas M, De Hert M, Hennekens $\mathrm{CH}$, Lambert M, Leucht S, Maj M, Mclntyre RS, Naber D, Newcomer JW, et al: Comorbid somatic illnesses in patients with severe mental disorders: clinical, policy, and research challenges. J Clin Psychiatry 2008, 69(4):514-519.

10. De Hert M, Cohen D, Bobes J, Cetkovich-Bakmas M, Leucht S, Ndetei DM, Newcomer JW, Uwakwe R, Asai I, Moller HJ, et al: Physical illness in patients with severe mental disorders. II. Barriers to care, monitoring and treatment guidelines, plus recommendations at the system and individual level. World Psychiatry 2011, 10(2):138-151.

11. Mitchell AJ, Lord O, Malone D: Differences in the prescribing of medication for physical disorders in individuals with $\mathrm{v}$. Without mental illness: meta-analysis. Br J Psychiatry 2012, 201(6):435-443.

12. Smith DJ, Langan J, McLean G, Guthrie B, Mercer SW: Schizophrenia is associated with excess multiple physical-health comorbidities but low levels of recorded cardiovascular disease in primary care: cross-sectional study. BMJ open 2013, 3(4):e002808. dx.doi.org/10.1136/bmjopen-2013-002808.

13. Thornicroft G, Rose D, Kassam A: Discrimination in health care against people with mental illness. Int Rev Psychiatry 2007, 19(2):113-122.

14. Harangozo J, Reneses B, Brohan E, Sebes J, Csukly G, Lopez-lbor J, Sartorius N, Rose D, Thornicroft G: Stigma and discrimination against people with schizophrenia related to medical services. Int J Soc Psychiatry 2013.

15. McManus $\mathrm{S}$, Meltzer $\mathrm{H}$, Campion J: Cigarette smoking and mental health in England. Data from the adult psychiatric morbidity survey. London: National Centre for Social Research; 2010.

16. Roick C, Fritz-Wieacker A, Matschinger H, Heider D, Schindler J, Riedel-Heller S, Angermeyer MC: Health habits of patients with schizophrenia. Soc Psychiatry Psychiatr Epidemiol 2007, 42(4):268-276.

17. von Hausswolff-Juhlin $Y$, Bjartveit $M$, Lindstrom E, Jones P: Schizophrenia and physical health problems. Acta psychiatrica Scandinavica Supplementum 2009, 438:15-21.

18. Chwastiak LA, Rosenheck RA, Kazis LE: Association of psychiatric illness and obesity, physical inactivity, and smoking among a national sample of veterans. Psychosomatics 2011, 52(3):230-236.

19. Vancampfort D, Correll CU, Probst M, Sienaert P, Wyckaert S, De Herdt A, Knapen J, De Wachter D, De Hert M: A review of physical activity correlates in patients with bipolar disorder. J Affect Disord 2013, 145(3):285-291.

20. Vancampfort D, Knapen J, Probst M, Scheewe T, Remans S, De Hert M: A systematic review of correlates of physical activity in patients with schizophrenia. Acta Psychiatrica Scand 2012, 125(5):352-362.

21. McDevitt J, Snyder M, Miller A, Wilbur J: Perceptions of barriers and benefits to physical activity among outpatients in psychiatric rehabilitation. J Nurs Scholarsh 2006, 38(1):50-55.

22. Suija K, Pechter U, Kalda R, Tahepold H, Maaroos J, Maaroos HI: Physical activity of depressed patients and their motivation to exercise: nordic walking in family practice. Int J Rehabil Res 2009, 32(2):132-138.

23. Rosenberg SD, Drake RE, Brunette MF, Wolford GL, Marsh BJ: Hepatitis C virus and HIV co-infection in people with severe mental illness and substance use disorders. AIDS 2005, 19(Suppl 3):S26-33.

24. Osher FC, Goldberg RW, McNary SW, Swartz MS, Essock SM, Butterfield MI, Rosenberg SD: Substance abuse and the transmission of hepatitis $C$ among persons with severe mental illness. Psychiatr Serv 2003, 54(6):842-847.

25. Chin CN, Sullivan K, Wilson SF: A 'snap shot' of the health of homeless people in inner Sydney: St Vincent's hospital. Aust Health Rev 2011 35(1):52-56.

26. Edidin JP, Ganim Z, Hunter SJ, Karnik NS: The mental and physical health of homeless youth: a literature review. Child Psychiatry Hum Dev 2012, 43(3):354-375 
27. Wahlbeck K, Westman J, Nordentoft M, Gissler M, Laursen TM: Outcomes of nordic mental health systems: life expectancy of patients with mental disorders. Br J Psychiatry 2011, 199(6):453-458

28. Chang CK, Hayes RD, Perera G, Broadbent MT, Fernandes AC, Lee WE, Hotopf M, Stewart R: Life expectancy at birth for people with serious mental illness and other major disorders from a secondary mental health care case register in London. PLOS One 2011, 6(5):e19590.

29. Osborn DP, Levy G, Nazareth I, Petersen I, Islam A, King MB: Relative risk of cardiovascular and cancer mortality in people with severe mental illness from the United Kingdom's general practice rsearch database. Arch Gen Psychiatry 2007, 64(2):242-249.

30. Saha S, Chant D, McGrath J: A systematic review of mortality in schizophrenia: is the differential mortality gap worsening over time? Arch Gen Psychiatry 2007, 64(10):1123-1131.

31. Centorrino F, Mark TL, Talamo A, Oh K, Chang J: Health and economic burden of metabolic comorbidity among individuals with bipolar disorder. J Clin Psychopharmacol 2009, 29(6):595-600.

32. Fagiolini A, Goracci A: The effects of undertreated chronic medical illnesses in patients with severe mental disorders. J Clin Psychiatry 2009, 70(Suppl 3):22-29.

33. Lehnert T, Konnopka A, Riedel-Heller S, Konig HH: Health economic aspects of physical-mental comorbidity. Bundesgesundheitsblatt Gesundheitsforschung Gesundheitsschutz 2011, 54(1):120-127.

34. Rajagopalan K, Kleinman NL, Brook RA, Gardner HH, Brizee TJ, Smeeding JE: Costs of physical and mental comorbidities among employees: a comparison of those with and without bipolar disorder. Curr Med Res Opin 2006, 22(3):443-452

35. Shen C, Sambamoorthi U, Rust G: Co-occurring mental illness and health care utilization and expenditures in adults with obesity and chronic physical illness. Dis Manag 2008, 11(3):153-160.

36. Molosankwe I, Patel A, Jose Gagliardino J, Knapp M, McDaid D: Economic aspects of the association between diabetes and depression: a systematic review. J Affect Disord 2012, 142(Suppl):S42-55.

37. Naylor C, Parsonage M, McDaid D, Knapp M, Fossey M, Galea A: Long-term conditions and mental health. The costs of co-morbdities. London: The King's Fund; 2012

38. Weiser P, Becker T, Losert C, Alptekin K, Berti L, Burti L, Burton A, Dernovsek M, Dragomirecka $E$, Freidl $M$, et al: European network for promoting the physical health of residents in psychiatric and social care facilities (HELPS): background, aims and methods. BMC Publ Health 2009, 9:315.

39. Weiser P: Catalogue of effectiveness and cost-effective interventions programmes. Ulm: University of Ulm; 2011:415-462.

40. Bartels S, Desilets R: Health promotion programs for people with serious mental illness (prepared by the dartmouth health promotion research team). Washington, D. C.: SAMHSA-HRSA Center for Integrated Health Solutions; 2012.

41. Verhaeghe N, De Maeseneer J, Maes L, Van Heeringen C, Annemans L: Effectiveness and cost-effectiveness of lifestyle interventions on physical activity and eating habits in persons with severe mental disorders: a systematic review. Int J Behav Nutr Phys Act 2011, 8:28.

42. Vancampfort D, Probst M, Helvik Skjaerven L, Catalan-Matamoros D, Lundvik-Gyllensten A, Gomez-Conesa A, ljntema R, De Hert M: Systematic review of the benefits of physical therapy within a multidisciplinary care approach for people with schizophrenia. Physical therapy 2012 92(1):11-23.

43. McDaid D, Park AL: Investing in mental health and well-being: findings from the DataPrev project. Health Promot Int 2011, 26(Suppl 1):i108-139.

44. Moher D, Liberati A, Tetzlaff J, Altman DG: Preferred reporting items for systematic reviews and meta-analyses: the PRISMA statement. PLOS medicine 2009, 6(7):e1000097.

45. Drummond MF, Schulpher MJ, Torrance GW, O'Brien BJ, Stoddart GL: Methods for the economic evaluation of health care programmes. Oxford: Oxford University Press; 2005

46. Timko C, Chen S, Sempel J, Barnett P: Dual diagnosis patients in community or hospital care: one-year outcomes and health care utilization and costs. J Ment Health 2006, 15(2):163-177.

47. Pinkerton SD, Johnson-Masotti AP, Otto-Salaj LL, Stevenson LY, Hoffmann RG Cost-effectiveness of an HIV prevention intervention for mentally ill adults. Ment Health Serv Res 2001, 3(1):45-55.

48. Johnson-Masotti AP, Pinkerton SD, Kelly JA, Stevenson LY: Costeffectiveness of an HIV risk reduction intervention for adults with severe mental illness. AIDS Care 2000, 12(3):321-332.
49. Gusi N, Reyes MC, Gonzalez-Guerrero JL, Herrera E, Garcia JM: Cost-utility of a walking programme for moderately depressed, obese, or overweight elderly women in primary care: a randomised controlled trial. BMC Publ Health 2008, 8:231.

50. Murphy SM, Edwards RT, Williams N, Raisanen L, Moore G, Linck P Hounsome N, Din NU, Moore L: An evaluation of the effectiveness and cost effectiveness of the national exercise referral scheme in Wales, UK: a randomised controlled trial of a public health policy initiative. J Epidemiol Community Health 2012, 66(8):745-753.

51. Chalder M, Wiles NJ, Campbell J, Hollinghurst SP, Searle A, Haase AM, Taylor AH, Fox KR, Baxter $\mathrm{H}$, Davis $\mathrm{M}$, et al: A pragmatic randomised controlled trial to evaluate the cost-effectiveness of a physical activity intervention as a treatment for depression: the treating depression with physical activity (TREAD) trial. Health Technol Assess 2012, 16(10):1-164. iii-iv.

52. Carter T, Callaghan P, Khalil E, Morres I: The effectiveness of a preferred intensity exercise programme on the mental health outcomes of young people with depression: a sequential mixed methods evaluation. BMC Publ Health 2012, 12:187.

53. Verhaeghe $\mathrm{N}$, De Maeseneer J, Maes $\mathrm{L}$, Van Heeringen C, Bogaert $\mathrm{V}$, Clays $\mathrm{E}$, De Bacquer D, Annemans L: Health promotion intervention in mental health care: design and baseline findings of a cluster preference randomized controlled trial. BMC Publ Health 2012, 12:431.

54. Kruisdijk FR, Hendriksen IJ, Tak EC, Beekman AT, Hopman-Rock M: Effect of running therapy on depression (EFFORT-D). design of a randomised controlled trial in adult patients [ISRCTN 1894]. BMC Publ Health 2012, 12:50.

55. Barnett $P G$, Wong W, Hall S: The cost-effectiveness of a smoking cessation program for out-patients in treatment for depression. Addiction 2008, 103(5):834-840.

56. Stockings EA, Bowman JA, Wiggers J, Baker AL, Terry M, Clancy R, Wye PM, Knight J, Moore LH: A randomised controlled trial linking mental health inpatients to community smoking cessation supports: a study protocol. BMC Publ Health 2011, 11:570.

57. Bonevski B, Paul C, D'Este C, Sanson-Fisher R, West R, Girgis A, Siahpush M, Carter R: RCT of a client-centred, caseworker-delivered smoking cessation intervention for a socially disadvantaged population. BMC Publ Health 2011, 11(1):70

58. Craig TK, Johnson S, McCrone P, Afuwape S, Hughes E, Gournay K, White I, Wanigaratne S, Leese M, Thornicroft G: Integrated care for co-occurring disorders: psychiatric symptoms, social functioning, and service costs at 18 months. Psychiatr Serv 2008, 59(3):276-282.

59. Morse GA, Calsyn RJ, Klinkenberg WD, Helminiak TW, Wolff N, Drake RE, Yonker RD, Lama G, Lemming MR, McCudden S: Treating homeless clients with severe mental illness and substance use disorders: costs and outcomes. Community Ment Health J 2006, 42(4):377-404

60. Rosenberg S, Brunette M, Oxman T, Marsh B, Dietrich A, Mueser K, Drake R, Torrey W, Vidaver R: The STIRR model of best practices for blood-borne diseases among clients with serious mental illness. Psychiatr Serv 2004, 55(6):660-664

61. Rosenberg SD, Goldberg RW, Dixon LB, Wolford GL, Slade EP, Himelhoch S, Gallucci G, Potts W, Tapscott S, Welsh CJ: Assessing the STIRR model of best practices for blood-borne infections of clients with severe mental illness. Psychiatr Serv 2010, 61(9):885-891.

62. Gold MR, Siegel JE, Russell LB, Weinstein MC: Cost-effectiveness in health and medicine. New York: Oxford University Press; 1996.

63. Baker A, Richmond R, Haile M, Lewin TJ, Carr VJ, Taylor RL, Jansons S, Wilhelm K: A randomized controlled trial of a smoking cessation intervention among people with a psychotic disorder. Am J Psychiatry 2006, 163(11):1934-1942.

64. Richardson CR, Avripas SA, Neal DL, Marcus SM: Increasing lifestyle physical activity in patients with depression or other serious mental illness. J Psychiatr Pract 2005, 11(6):379-388.

65. Tosh G, Clifton A, Mala S, Bachner M: Physical health care monitoring for people with serious mental illness. Cochrane Database Syst Rev 2010, 3, CD008298.

66. Hall SM, Prochaska JJ: Treatment of smokers with co-occurring disorders: emphasis on integration in mental health and addiction treatment settings. Annu Rev Clin Psychol 2009, 5:409-431.

67. Coleman T, Agboola S, Leonardi-Bee J, Taylor M, McEwen A, McNeill A Relapse prevention in UK stop smoking services: current practice, systematic reviews of effectiveness and cost-effectiveness analysis. Health Technol Assess 2010, 14(49):1-152. iii-iv. 
68. Salize HJ, Merkel S, Reinhard I, Twardella D, Mann K, Brenner H: Cost-effective primary care-based strategies to improve smoking cessation: more value for money. Arch Intern Med 2009, 169(3):230-235. discussion 235-236.

69. McDaid D, Needle J: What use has been made of economic evaluation in public health? a systematic review of the literature. In The future of health: burdens, challenges, and opportunities. Edited by Morris Z, Dawson S. Basingstoke: Palgrave McMillan; 2009.

70. Tidey JW: Using incentives to reduce substance use and other health risk behaviors among people with serious mental illness. Preventive medicine 2012, 55(Suppl):S54-60.

71. Chivers LL, Higgins ST: Some observations from behavioral economics for consideration in promoting money management among those with substance use disorders. Am J Drug Alcohol Abuse 2012, 38(1):8-19.

72. Roberts SH, Bailey JE: Incentives and barriers to lifestyle interventions for people with severe mental illness: a narrative synthesis of quantitative, qualitative and mixed methods studies. J Adv Nurs 2011, 67(4):690-708.

73. Jormfeldt $\mathrm{H}$ : Supporting positive dimensions of health, challenges in mental health care. International journal of qualitative studies on health and well-being 2011, 6(2):7126. dx.doi.org/10.3402/qhw.v6i2.7126.

74. McDaid D, Sassi F: Equity, efficiency and research synthesis. In Evidencebased decisions and economics: health care, social welfare, education and criminal justice second edition. Edited by Shemilt I, Mugford M, Vale L, Marsh K, Donaldson C. Oxford: Wiley-Blackwell; 2010.

75. Caspi CE, Kawachi I, Subramanian SV, Adamkiewicz G, Sorensen G: The relationship between diet and perceived and objective access to supermarkets among low-income housing residents. Soc Sci Med 2012, 75(7):1254-1262

76. Lorenc T, Petticrew M, Welch V, Tugwell P: What types of interventions generate inequalities? evidence from systematic reviews. J Epidemiol Community Health 2013, 67(2):190-193.

77. Kilian R, Matschinger H, Loeffler W, Roick C, Angermeyer MC: A comparison of methods to handle skew distributed cost variables in the analysis of the resource consumption in schizophrenia treatment. J Ment Health Policy Econ 2002, 5(1):21-31.

78. Kelly MP, McDaid D, Ludbrook A, Powell J: Economic appraisal of public health interventions. London: Health Development Agency; 2005.

79. Kasteng F, Eriksson J, Sennfalt K, Lindgren P: Metabolic effects and costeffectiveness of aripiprazole versus olanzapine in schizophrenia and bipolar disorder. Acta Psychiatr Scand 2011, 124(3):214-225.

80. Park A, McCrone P, Knapp M: Early intervention for first episode psychosis: economic impacts within and beyond the health care system, a decision modelling approach. London: Personal Social Services Research Unit; 2012.

doi:10.1186/1471-2458-13-787

Cite this article as: Park et al: Examining the cost effectiveness of interventions to promote the physical health of people with mental health problems: a systematic review. BMC Public Health 2013 13:787.

\section{Submit your next manuscript to BioMed Central and take full advantage of:}

- Convenient online submission

- Thorough peer review

- No space constraints or color figure charges

- Immediate publication on acceptance

- Inclusion in PubMed, CAS, Scopus and Google Scholar

- Research which is freely available for redistribution 